\title{
Does joint line elevation after revision knee arthroplasty affect tibio-femoral kinematics, contact pressure or collateral ligament lengths? An in vitro analysis
}

Jacek B. Kowalczewski ${ }^{1}$, Luc Labey², Yan Chevalier ${ }^{3}$, Tomasz Okon ${ }^{1}$, Bernardo Innocenti ${ }^{4}$, Johan Bellemans ${ }^{5}$

\begin{abstract}
${ }^{1}$ Department of Orthopaedics and Inflammatory Disorders of Locomotor System, The Medical Centre of Postgraduate Education, Otwock, Poland

${ }^{2} \mathrm{KU}$ Leuven, Mechanical Engineering Technology TC Geel, Geel, Belgium

${ }^{3}$ Labor für Biomechanik und Experimentelle Orthopädie, Orthopädische Klinik und Poliklinik, Klinikum Großhadern, München, Germany

${ }^{4}$ Bio Electro and Mechanical Systems Department, Université Libre de Bruxelles, Brussel, Belgium

${ }^{5}$ Department of Orthopaedic Surgery and Traumatology, Ziekenhuis Oost Limburg, Genk, Belgium
\end{abstract}

Submitted: 8 April 2013

Accepted: 7 May 2013

Arch Med Sci 2015; 11, 2: 311-318

DOI: 10.5114 /aoms.2014.46078

Copyright @ 2015 Termedia \& Banach

\section{Abstract}

Introduction: Correct restoration of the joint line is generally considered as crucial when performing total knee arthroplasty (TKA). During revision knee arthroplasty however, elevation of the joint line occurs frequently. The general belief is that this negatively affects the clinical outcome, but the reasons are still not well understood.

Material and methods: In this cadaveric in vitro study the biomechanical consequences of joint line elevation were investigated using a previously validated cadaver model simulating active deep knee squats and passive flexion-extension cycles. Knee specimens were sequentially tested after total knee arthroplasty with joint line restoration and after $4 \mathrm{~mm}$ joint line elevation.

Results: The tibia rotated internally with increasing knee flexion during both passive and squatting motion (range: $17^{\circ}$ and $7^{\circ}$ respectively). Joint line elevation of $4 \mathrm{~mm}$ did not make a statistically significant difference. During passive motion, the tibia tended to become slightly more adducted with increasing knee flexion (range: $2^{\circ}$ ), while it went into slighlty less adduction during squatting (range: $-2^{\circ}$ ). Neither of both trends was influenced by joint line elevation. Also anteroposterior translation of the femoral condyle centres was not affected by joint line elevation, although there was a tendency for a small posterior shift (of about $3 \mathrm{~mm}$ ) during squatting after joint line elevation. In terms of kinetics, ligaments lengths and length changes, tibiofemoral contact pressures and quadriceps forces all showed the same patterns before and joint line elevation. No statistically significant changes could be detected. Conclusions: Our study suggests that joint line elevation by $4 \mathrm{~mm}$ in revision total knee arthroplasty does not cause significant kinematic and kinetic differences during passive flexion/extension movement and squatting in the tibio-femoral joint, nor does it affect the elongation patterns of collateral ligaments. Therefore, clinical problems after joint line elevation are probably situated in the patello-femoral joint or caused by joint line elevation of more than $4 \mathrm{~mm}$.
Corresponding author: Prof. Luc Labey European Centre for Knee Research, Smith and Nephew Researchpark Haasrode 1030 Technologielaan 11 bis 3001 Leuven, Belgium Phone: +32477973658 E-mail: luc.labey@mech. kuleuven.be

Key words: revision, kinematics, contact pressure, collateral ligaments. 


\section{Introduction}

Optimal knee joint function requires a delicate balance between the osseous anatomy and the surrounding soft tissues. Balancing the collateral ligaments is therefore considered as an important factor affecting the clinical success after total knee arthroplasty (TKA) [1]. Leaving the knee too loose may theoretically lead to tibio-femoral instability, whereas excessive tightness may cause stiffness [2-6]. Elevation of the knee joint line during TKA could in theory disturb this balance and should therefore probably be avoided.

Joint line elevation is nevertheless relatively common, especially during revision TKA. Partington [7] reported joint line elevation in $79 \%$ of revision TKAs. A natural tendency indeed exists for the surgeons to proximalise the joint line in revision TKA [7-9]. Today, it is not fully clear what the consequences are of joint line elevation on the post-operative performance after TKA. Although several studies have found no correlation between joint line elevation and clinical outcome [8, 10, $11]$, others have linked an elevated joint line to inferior clinical and functional results [7, 12-16]. To our knowledge, there are only a few studies which have evaluated the biomechanical consequences of joint line elevation [17].

From a biomechanical perspective, joint line elevation will first of all change the positions of the insertion regions of the collateral ligaments with respect to the flexion axes of the knee. As such, it will lead to deviations from the generally isometric behavior of the collateral ligaments [18, 19], but in a more complex way than simply loosening or tightening them. The effect on collateral ligament length will be dependent on the flexion angle. Secondly, joint line elevation will also affect the efficiency of the quadriceps mechanism because it causes a patella baja and it is generally accompanied by downsizing of the femoral component, which leads to a reduction of the quadriceps moment arm.

Consequently, understanding and quantifying the effects of joint line elevation during passive flexion-extension movement as well as during active motor tasks is an important step towards comprehending the role that restoration of the joint line has on clinical outcome. The aim of this study was therefore to analyze the influence of joint line elevation on tibio-femoral kinematics, collateral ligaments length patterns, tibio-femoral contact pressures and quadriceps efficiency. Thus, five fresh frozen human cadaver knees were tested in both passive (unloaded) flexion-extension cycles and loaded squats, both after primary TKA, and subsequently again after $4 \mathrm{~mm}$ elevation of the joint line in a revision procedure.
We hypothesized that joint line elevation would affect tibio-femoral kinematics mainly in the anteroposterior direction and in terms of tibial axial rotation. We also expected to see altered length patterns in the medial and lateral collateral ligaments, increased quadriceps forces and, as a consequence, increased tibio-femoral contact pressures.

\section{Material and methods}

The methodology for this study was identical to a previously published in vitro experiment [20,21]. Five cadaver knees were scanned using computed tomography (CT) prior to the experiments and with frames with four infrared reflective markers rigidly attached to the femur and tibia. CT images were analyzed with commercial medical image processing software (Mimics 11.02, Materialise, Haasrode, Leuven, Belgium) to identify ligament insertions and bony landmarks. The limb was sectioned $32 \mathrm{~cm}$ cranial and $28 \mathrm{~cm}$ caudal to the tibio-femoral joint line. The femur and tibia were rigidly fixed with polymethylmethacrylate in containers, and the quadriceps, biceps femoris, and semimembranous and semitendinosus tendons were dissected and clamped.

All cadaver knees were opened and a posterior stabilized revision TKA prosthesis with a conventional type insert (Legion Revision, Smith \& Nephew, Memphis, TN, USA) was implanted according to the manufacturer's instructions, using conventional instrumentation. Femoral and tibial components were fixed with bone cement, and the appropriate conventional tibial polyethylene spacer was inserted with the thickness according to the spacer blocks. Patellae were left unresurfaced. Pressure sensitive films (I-Scan with Sensor model 4000, Tekscan, Inc, South Boston, MA, USA) were inserted and fixed to the tibial inserts using double-sided tape, which allowed measurement of tibio-femoral contact forces and pressures during motion.

The specimen was then mounted on a custom-made dynamic knee simulator system, based on the Oxford Rig, to simulate and record motions and loads during squatting (Figure 1). Five calibrated infrared cameras (Vicon Motion Systems, Los Angeles, CA, USA) recorded the motion of the femur and tibia through the reflective markers.

The implanted knee was then tested in passive and squat motion and its kinematics measured with the optical system. First, a passive test was performed with the femur mounted on the rig while the tibia was unconstrained and manually moved in flexion/extension. The tibia container was then mounted on the rig and a squat was performed by downwards displacement of the hip joint of the system while a programmed real-time 
control loop (Labview, National Instruments, Austin, TX, USA) maintained a constant $140 \mathrm{~N}$ ankle load. Hamstrings were pulled with constant springs $(50 \mathrm{~N})$ on the medial and lateral side.

Next, the femoral component was carefully removed, and the joint line was elevated by cutting the distal part of the femur by an extra $4 \mathrm{~mm}$. A one size smaller femoral component was then implanted using the same anterior cut in order to obtain similar flexion and extension gaps. Since the difference between two sizes in the Legion revision system is $3.5 \mathrm{~mm}$, the resulting flexion extension discrepancy increased by approximately $0.5 \mathrm{~mm}$, which we considered negligible. A $4 \mathrm{~mm}$ thicker polyethylene spacer was inserted, the pressure-sensitive film reattached, and the knee was tested again during passive flexion-extension and loaded deep knee squatting.

In a final step, ligament insertion points which were not visible on the CT scan were identified using a wand with five reflective markers and the same camera system used to record the kinematics.

Using the superimposed CT bony landmarks and the relative motion of the optical markers, 3D kinematics of the knee joint and ligament elongation patterns were calculated based on coordinate systems and joint rotations proposed by Grood and Suntay [22]. For all specimens, the passive and squat kinematics and elongation patterns of the superficial medial and lateral collateral ligaments (SMCL and LCL) were recorded and compared before and after joint line elevation. Quadriceps forces were also measured and compared during squat tests. For comparison purposes, all kinematic data were resampled at 10-degree intervals of knee flexion angle, and restricted to the common range of flexion for each test type for all specimens. Means and standard deviations were then calculated at each flexion angle increment of the common range of motion. In addition to standard kinematic indices (tibial internal rotation, adduction, flexion angle, antero-posterior and medio-lateral translations), displacements were also expressed in terms of the femoral lateral and medial condyle centers (FLCC and FMCC) projected on the tibial plateau and normalized to the antero-posterior tibial plateau width. Maximal tibio-femoral contact pressures were compared for the two TKA situations during the squatting motion.

\section{Statistical analysis}

Statistical paired $T$-tests were finally carried out to detect significant differences in kinematic patterns, ligament elongation, tibio-femoral contact pressures and quadriceps forces between the primary TKA and after joint line elevation by $4 \mathrm{~mm}$.

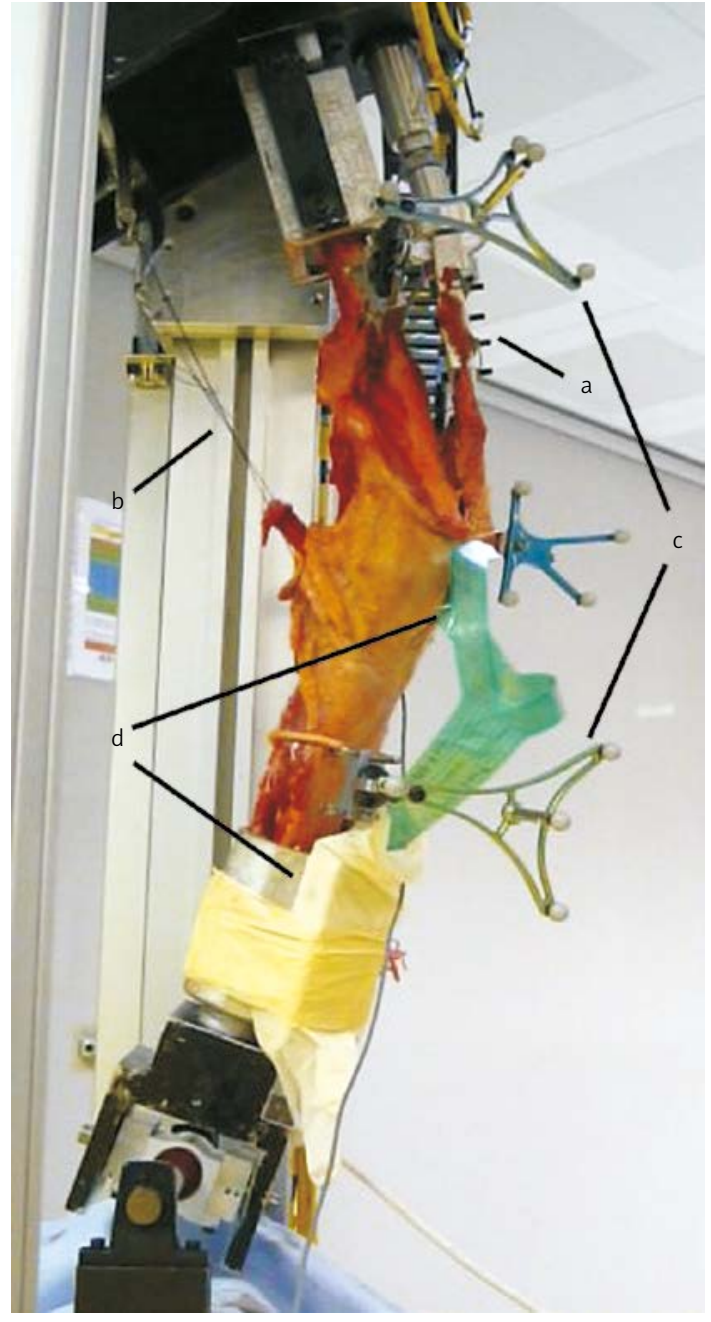

Figure 1. The experimental set-up with a cadaver specimen mounted on the knee rig, ready for a squat. The rig allows $6^{\circ}$ of freedom for the knee joint. Visible are the clamp fixed to the quadriceps tendon (a) and cables attached to medial and lateral hamstring tendons (b); the marker frames on tibia and femur (c) and the Tekscan sensor and handle (d)

\section{Results}

\section{Tibio-femoral kinematics}

Tibial external rotation and adduction kinematics are shown in Figure 2 for passive and squat motion after primary TKA and after joint line elevation by $4 \mathrm{~mm}$. The common ranges of flexion angle were $20-120^{\circ}$ and $30-90^{\circ}$ for passive and squat motions, respectively. For passive flexionextension cycles, tibial external rotation and adduction were statistically similar before and after joint line elevation. Squatting motion further revealed no statistically significant changes in tibial external rotation and adduction for the whole range of motion between primary TKA and after joint line elevation by $4 \mathrm{~mm}$. 


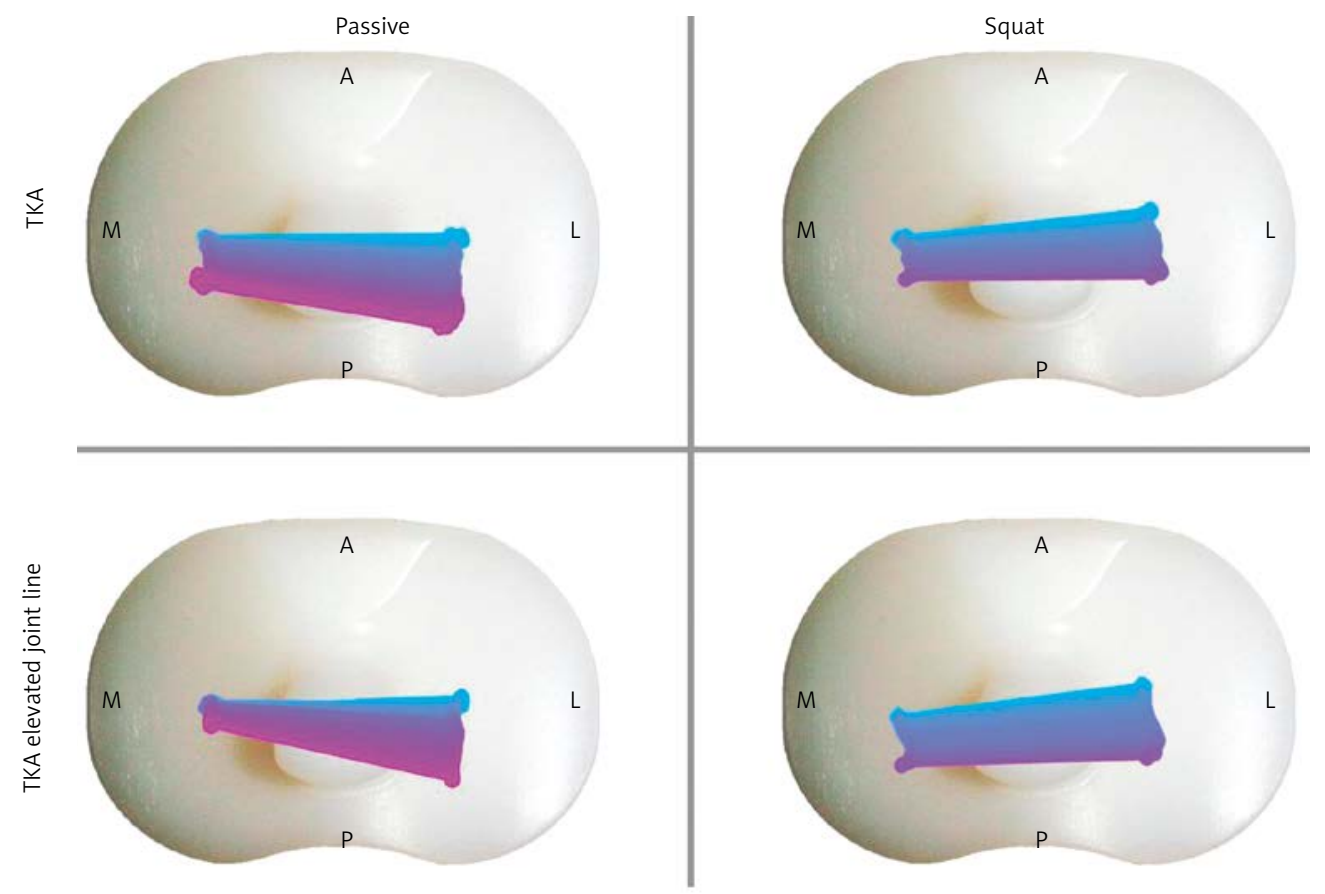

Flexion angle $\left({ }^{\circ}\right)$

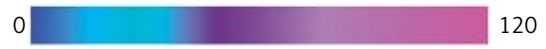

Figure 2. Top view of the tibial insert with the medial and lateral femoral condyle centers projected onto it during the different motion cycles (passive cycles on the left, squats on the right). Top row shows knee kinematics after primary TKA with joint line reconstruction, lower row shows knee kinematics after revision TKA with $4 \mathrm{~mm}$ joint line elevation

$M$ - medial, $L$ - lateral, $A$-anterior, $P$ - posterior.

No significant changes were noted in the displacements of the femoral condyle centers relative to the tibial plateau before and after joint line elevation (Figure 3). In passive flexion, joint line elevation decreased the posterior translation of the projected femoral medial condyle center, especially beyond $40^{\circ}$ of flexion. A slight $5 \%$ posterior shift of the FMCC and FLCC was noted after joint line elevation during squatting (corresponding to $3 \mathrm{~mm}$ in a typical tibia of about $6 \mathrm{~cm}$ anteroposterior size). This difference was, however, not statistically significant.

\section{Collateral ligament elongation patterns}

The length of the collateral ligaments prior to implantation of the prosthesis was used as the baseline. Length measurements show that the sMCL and LCL length remained mostly constant during passive flexion in the TKA knees, and were unaffected by elevation of the joint line (Figure 4). During squatting, the SMCL lengthened with flexion after primary TKR but stayed constant when the joint line was elevated. The LCL length change during the flexion phase of the squat showed somewhat more shortening after joint line elevation at higher flexion, but this difference was not statistically significant.

\section{Changes in tibio-femoral contact pressures and quadriceps forces}

Tibio-femoral joint kinetics were observed not to be affected by a $4 \mathrm{~mm}$ elevation of the joint line. In particular, while mean contact pressures after joint line elevation were $40 \%$ higher than primary TKA in the initial $30-40^{\circ}$ of flexion, these differences were not statistically significant and gradually diminished for higher flexion angles (Figure $5 \mathrm{~A}$ ). The results also showed no significant differences in mean quadriceps loads between primary TKA and after raising the joint line (Figure 5 B).

\section{Discussion}

Although there is some clinical evidence that elevation of the joint line may be associated with inferior clinical results $[7,9,13,14,16]$, the effects of joint line elevation on knee biomechanics remain relatively unknown. In this study we specifically evaluated these effects on tibio-femoral kinematics and kinetics, elongation patterns of the collateral ligaments, as well as quadriceps load in an in vitro set-up. However, we could not detect any statistically significant effects caused by the 

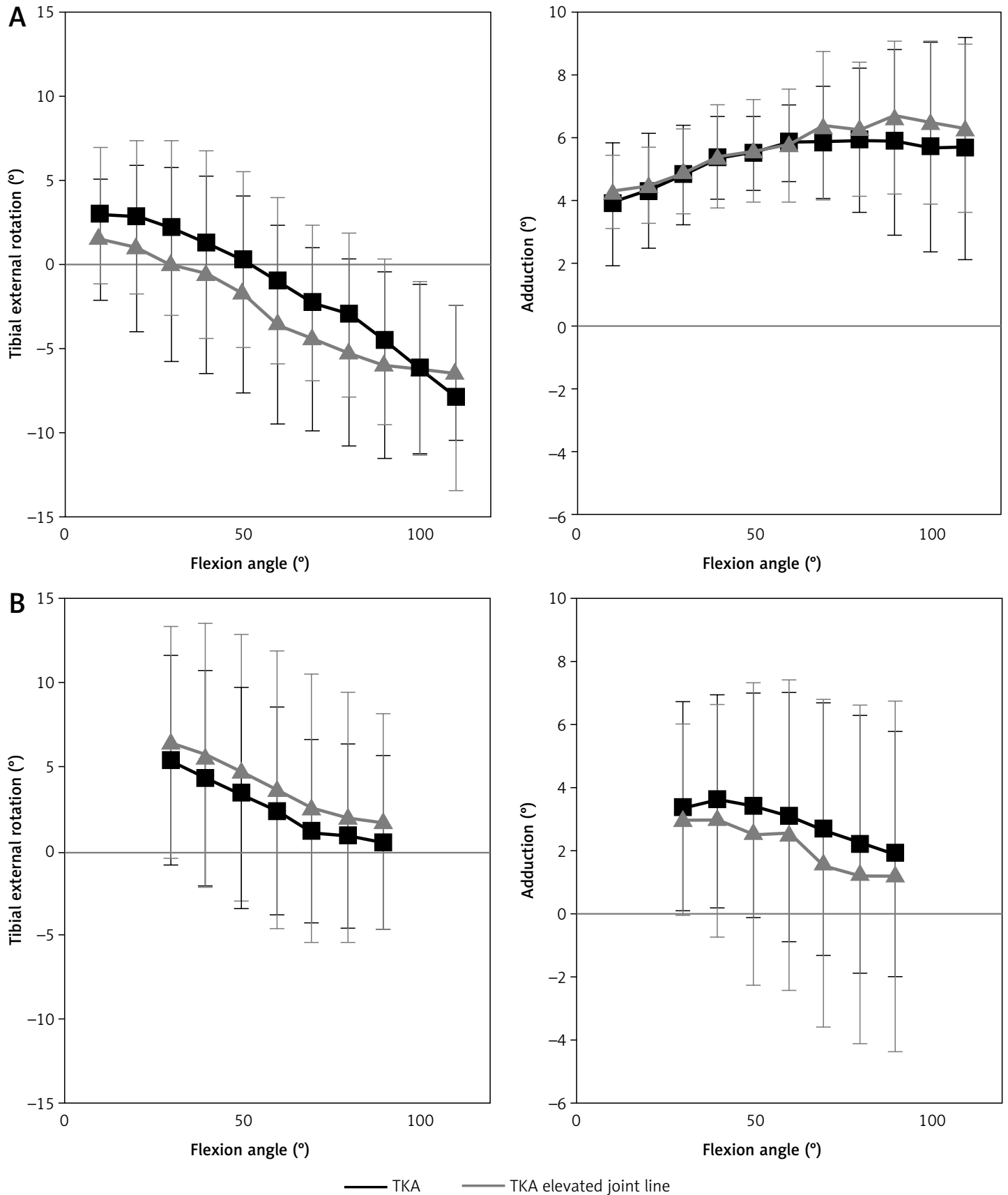

Figure 3. Tibial external rotation and tibial adduction as a function of flexion angle during passive flexion-extension cycles (A) and during squatting (B)

induced joint line elevation for any of the investigated parameters.

Regarding kinematics, posterior translation of both FMCC and FLCC was observed for the implanted knees. In passive motion, the FMCC showed less posterior translation than the FLCC, which is similar to the motion patterns for the intact knee joint as observed in the literature [20]. This effect was more pronounced after joint line elevation, with an almost stationary FMCC. During squatting, both condyles translated posteriorly in almost the same amount for primary TKA, with only a slight posterior but not significant shift occurring after joint line elevation.

Existing reports link joint line elevation to observed mid flexion instability [15]. In order to raise the joint line in our study, the distal cut of the femur was proximalized by $4 \mathrm{~mm}$. A one size smaller femoral component was implanted to equalize the flexion and extension gap. Consequently, after the joint line elevation procedure, using a $4 \mathrm{~mm}$ thicker polyethylene insert, flexion and extension joint gaps were almost equal. In passive motion, our results showed no significant differences of 

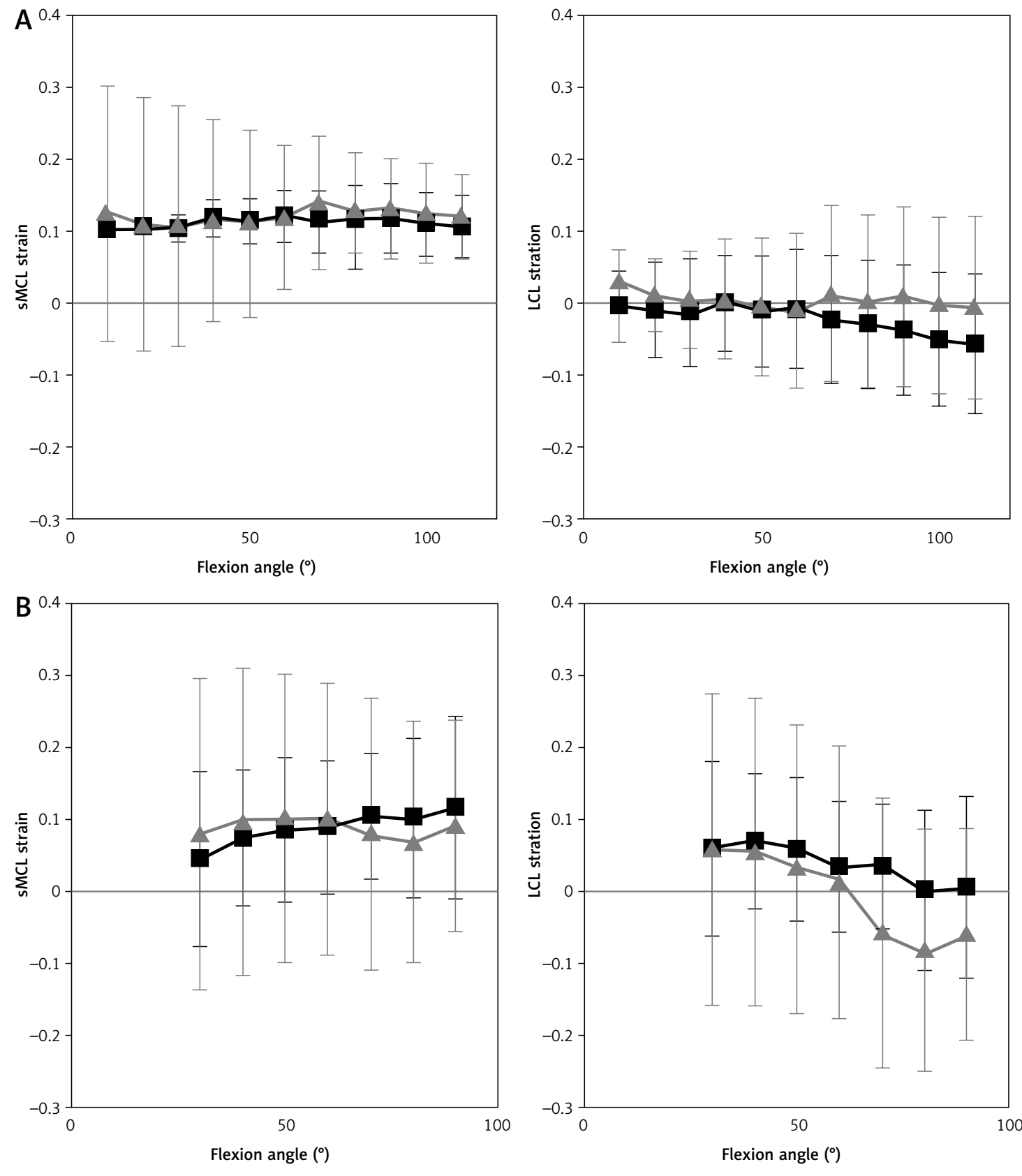

Figure 4. Relative length changes of $S M C L$ and $L C L$ with respect to their initial, native length as a function of flexion angle during passive flexion-extension cycles (A) and during squatting (B)

joint line elevation for collateral ligaments length patterns, which is consistent with reports from Jeffcote, who reported no influence of differences in flexion and extension gaps on collateral ligament length changes during passive movement [23]. Our results further showed no significant effect of joint line elevation on collateral ligament lengths during loaded squatting, and did not show any potential for midflexion instability in active motion, which was further supported by the lack of statistically significant differences in varus-valgus stability before and after joint line elevation.
Regarding joint kinetics, tibio-femoral contact pressure measurements showed no differences after elevation of the joint line. This is consistent with the numerical predictions of König et al. [17]. The latter reported that elevating the joint line by $10 \mathrm{~mm}$ - thus more than twice as much as the current study - only slightly increased tibio-femoral contact forces in stair climbing [17] compared to an anatomical TKA. These authors instead predicted increased patello-femoral contact forces, and suggested that the main biomechanical effect of raising the joint line in revision TKA may be due 

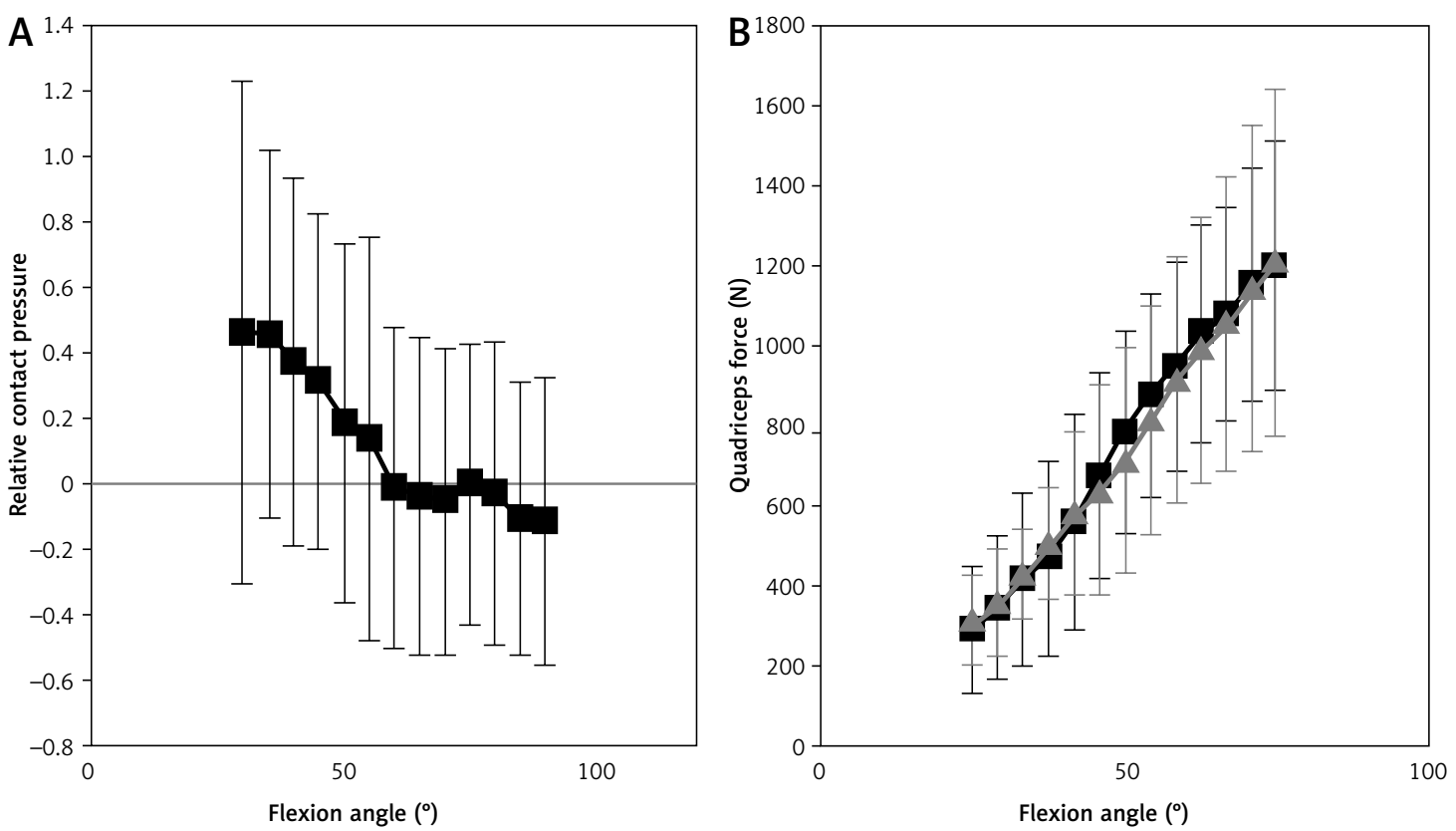

Figure 5. Change in tibio-femoral contact pressure after $4 \mathrm{~mm}$ joint line elevation with respect to contact pressure after primary TKA as a function of flexion angle during squatting (A) and quadriceps force as a function of flexion angle during squatting (B)

to increases in patella-femoral contact loads. Their hypothesis was not explicitly verified in the current study, but as our results showed no differences in quadriceps forces after joint line elevation, it is possible that such effects may be observable for TKA revisions involving joint line elevations which are higher than the $4 \mathrm{~mm}$ level used in the current study.

In a clinical study, Figgie et al. reported that joint line elevation correlated with lower functional knee scores, patello-femoral pain, and the need for revision [13]. As our current study did not detect any effect of joint line elevation on tibio-femoral kinematics, kinetics, or length patterns of the collateral ligaments, it is possible that in revision TKA, these effects may be limited to or triggered at the patello-femoral joint, or only become significant with higher joint line elevations than the $4 \mathrm{~mm}$ level tested in the current study. This hypothesis is currently being investigated in a new in vitro study.

Some limitations may be raised for the current study.

First, only a limited number of five specimens was tested, and a larger sample size could have revealed more significant differences. Nevertheless, post hoc analysis showed that our study was strong enough in power to detect a difference of $12 \%$ in the collateral ligament length pattern if such a difference truly exists.

Secondly, only one implant system was tested and, consequently, the results and conclusions are only applicable to the implant under investigation. Other implants, with different J-curves, post-cam mechanisms, and articular shapes, may well show different behavior.

Thirdly, the accuracy of the optical tracking system which was used to measure joint motion might not be sufficient to detect small differences in kinematic pathways. However, we have found that our methodology and technique are sufficiently accurate and precise to detect differences in translations (and lengths) and rotations of less than $2 \mathrm{~mm}$ and $2^{\circ}$ respectively [21].

Fourthly, active motions were limited to a squat induced by a limited amount of simulated muscle loads, with hamstrings of constant and equal forces. While additional muscle actions could have been useful in evaluating more thoroughly the biomechanical effects of joint line elevation, a squatting motion was deemed sufficient as it involves high forces through a large range of motion.

A final limitation comes from the fact that only the $4 \mathrm{~mm}$ joint line elevation configuration was performed in the current study. Greater joint line elevation might have revealed more significant differences in knee biomechanics. They were however not considered as they might not be representative of typical revisions. Moreover, even if clinical problems only show up after higher amounts of joint line elevation, one might expect to already measure subtle differences in knee behavior, especially with the sensitivity and precision which can be obtained with the used equipment and 
methodology. Also, a rise of the joint line of $8 \mathrm{~mm}$ would be incompatible with the existing insert thicknesses of the Legion system.

In conclusion, our study suggests that joint line elevation in revision total knee arthroplasty does not cause significant kinematic and kinetic differences during passive flexion/extension movement and squat in the tibio-femoral joint, nor does it affect the elongation patterns of collateral ligaments. Further studies are necessary (and are in fact ongoing) to understand the reasons for inferior clinical outcomes related to the elevated joint line, more particularly related to the patello-femoral joint.

\section{Acknowledgments}

This work was financially supported by Smith and Nephew and performed at the European Centre for Knee Research, Smith \& Nephew, Researchpark Haasrode 1030, Technologielaan 11 Bis, 3001 Leuven, Belgium. The authors gratefully acknowledge Ronny De Corte's contribution to the study.

Each author certifies that he has no commercial associations (e.g., consultancies, stock ownership, equity interest, patent/licensing arrangements) that might pose a conflict of interest in connection with the submitted article.

\section{Conflict of interest}

The authors declare no conflict of interest.

\section{References}

1. Insall JN, Easly ME. Surgical techniques and instrumentation in total knee arthroplasty. In: Surgery of the knee. Insall JN, Scott WN (eds.). Churchill Livingstone, New York 2001; 1553-620.

2. Fehring TK, Valadie AL. Knee instability after total knee arthroplasty. Clin Orthop Relat Res 1994; 299: 157-62.

3. Keeney JA, Clohisy JC, Curry M, Maloney WJ. Revision total knee arthroplasty for restricted motion. Clin Orthop Relat Res 2005; 440: 135-40.

4. Kelly MA, Clarke HD. Stiffness and ankylosis in primary total knee arthroplasty. Clin Orthop Relat Res 2003; 416: 68-73.

5. Kuster MS, Bitschnau B, Votruba T. Influence of collateral ligament laxity on patient satisfaction after total knee arthroplasty: a comparative bilateral study. Arch Orthop Trauma Surg 2004; 124: 415-7.

6. Yercan HS, Ait Si Selmi T, Sugun TS, Neyret P. Tibiofemoral instability in primary total knee replacement: a review. Part 1: Basic principles and classification. Knee 2005; 12: 257-66.

7. Partington PF, Sawhney J, Rorabeck CH, Barrack RL, Moore J. Joint line restoration after revision total knee arthroplasty. Clin Orthop Relat Res 1999; 367: 165-71.

8. Bellemans J. Restoring the joint line in revision TKA: does it matter? Knee 2004; 11: 3-5.

9. Laskin RS. Joint line position restoration during revision total knee replacement. Clin Orthop Relat Res 2002; 404: 169-71.
10. Ritter MA, Montgomery TJ, Zhou H, Keating ME, Faris PM, Meding JB. The clinical significance of proximal tibial resection level in total knee arthroplasty. Clin Orthop Relat Res 1999; 360: 174-81.

11. Selvarajah E, Hooper G. Restoration of the joint line in total knee arthroplasty. J Arthroplasty 2009; 24: 1099-102.

12. Chiu KY, Ng TP, Tang WM, Yau WP. Knee flexion after total knee arthroplasty. J Orthop Surg (Hong Kong) 2002; 10: 194-202.

13. Figgie HE, Goldberg VM, Heiple KG, Moller HS, Gordon NH. The influence of tibial-patellofemoral location on function of the knee in patients with the posterior stabilized condylar knee prosthesis. J Bone Joint Surg Am 1986; 68: 1035-40.

14. Laskin RS. Management of the patella during revision total knee replacement arthroplasty. Orthop Clin North Am 1998; 29: 355-60.

15. Martin JW, Whiteside LA. The influence of joint line position on knee stability after condylar knee arthroplasty. Clin Orthop Relat Res 1990; 259: 146-56.

16. Porteous AJ, Hassaballa MA, Newman JH. Does the joint line matter in revision total knee replacement? J Bone Joint Surg Br 2008; 90-B: 879-84.

17. König C, Sharenkov A, Matziolis G, et al. Joint line elevation in revision TKA leads to increased patellofemoral contact forces. J Orthop Res 2010; 28: 1-5.

18. Victor J, Wong P, Witvrouw E, Vander Sloten J, Bellemans J. How isometric are the medial patellofemoral, superficial medial collateral, and lateral collateral ligaments of the knee? Am J Sports Med 2009; 37: 2028-36.

19. Kuster MS, Jeffcote BO, Schirm AC, Jacob H, Nicholls RL. Assessment of isometricity before and after total knee arthroplasty: a cadaver study. Knee 2009; 16: 352-7.

20. Victor J, Labey L, Wong P, Innocenti B, Bellemans J. The influence of muscle load on tibiofemoral knee kinematics. J Orthop Res 2010; 28: 419-28.

21. Victor J, Van Glabbeek F, Vander Sloten J, Parizel PM, Somville J, Bellemans J. An experimental model for kinematic analysis of the knee. J Bone Joint Surg Am 2009; 91 (Suppl. 6): 150-63.

22. Grood ES, Suntay WJ. A joint coordinate system for the clinical description of three-dimensional motions: application to the knee. J Biomech Eng 1983; 105: 136-44.

23. Jeffcote B, Nicholls R, Schirm A, Kuster MS. The variation in medial and lateral collateral ligament strain and tibiofemoral forces following changes in the flexion and extension gaps in total knee replacement: a laboratory experiment using cadaver knees. J Bone Joint Surg $\mathrm{Br}$ 2007; 89-B: 1528-33. 